BS. Glutamate metabotropic receptors as targets for drug therapy in epilepsy. Eur J Pharmacol. 2003;476(1-2):3-16.

9. Tanaka K, et al. Epilepsy and exacerbation of brain injury in mice lacking the glutamate transporter
GLT-1. Science. 1997;276(5319):1699-1702.

10. Tian GF, et al. An astrocytic basis of epilepsy. Nat Med. 2005;11(9):973-981.

11. Chapman AG. Glutamate and epilepsy. J Nutr. 2000;130(4S Suppl):1043S-1045S.
12. Kong Q, Takahashi K, Schulte D, Stouffer N, Lin $\mathrm{Y}$, Lin CL. Increased glial glutamate transporter EAAT2 expression reduces epileptogenic processes following pilocarpine-induced status epilepticus. Neurobiol Dis. 2012;47(2):145-154

\title{
Working toward immune tolerance in lung transplantation
}

\author{
Xinguo Jiang and Mark R. Nicolls
}

VA Palo Alto Health Care System, Palo Alto, California, USA. Stanford University, Stanford, California, USA.

\begin{abstract}
Long-term allograft survival is a major challenge facing solid organ transplantation. Recent studies have shown a negative correlation between infiltration of memory $T$ cells and allograft survival. Furthermore, blockade of leukocyte activation increases acceptance of transplanted organs, including heart, liver, and kidney. Lung allografts are associated with high rates of rejection, and therapies that increase acceptance of other transplanted organs have not translated into the lung. In this issue of the JCI, Krupnick and colleagues demonstrate in a murine model that lung allograft acceptance requires infiltration of a specific $T$ cell population into the graft. This study highlights the unique immunobiology of the lung and the complexity of lung transplant tolerance.
\end{abstract}

Lungs have evolved over the millennia by adapting to the continual bombardment of foreign antigens, inhaled from the breathable environment, with a unique capacity to dampen overly exuberant immune responses. Lung physiology is distinct compared with more sterile compartments in the body, like the peritoneum (1), which are not constantly immunologically challenged. Despite the propensity of the lungs to dampen the immune responses, achieving immune tolerance is a particularly elusive goal for pulmonary transplant recipients. While there are examples of successful steroid withdrawal in kidney and liver transplant recipients $(2,3)$, examples of liver transplant recipients able to come off all immunosuppression over time (4), and examples of successful tolerance induction protocols $(5,6)$, similar examples are vanishingly rare among lung transplant recipients. A compounding issue is that the bronchial artery circulation, which normally supports airways with high- $\mathrm{O}_{2}$ blood, is not restored at the time of transplantation. Lack of circulation

Conflict of interest: The authors have declared that no conflict of interest exists.

Citation for this article: J Clin Invest. 2014; 124(3):967-970. doi:10.1172/JCI74701. potentially decreases the ability of grafted lungs to fend off pathogens and cope with immune injury (7). The inherent antigenicity of the lung and its lymphatic drainage into bronchus-associated lymphoid tissue (BALT) $(8,9)$ appear to be coupled with an imperfect barrier defense against pathogen invasion. Together, these qualities ultimately render the lung particularly difficult to target for durable tolerance. To overcome these inherent obstacles to organ health, more information is clearly needed about the unique immunobiology of the transplanted lung.

In this issue of the JCI, Krupnick and colleagues (10) evaluated immune responses in murine orthotopic lung transplant recipients that were treated with costimulation blockade and specifically addressed how memory $\mathrm{T}$ lymphocytes contribute to the difficulty of achieving immune tolerance. Krupnick et al. discovered a population of memory $\mathrm{CD}^{+} \mathrm{T}$ cells that were paradoxically protective for the grafted lung through their regulatory function. These results further emphasize the unique complexity of pulmonary-specific transplant responses, which must be further evaluated if progress is to be made toward generating clinically important therapies to preserve protective immunity.

\section{Immune tolerance and acute rejection in lung transplantation}

Immune tolerance can be defined in several ways. In robust animal models, specific immune tolerance refers to an antigenspecific unresponsive immunologic state that preserves responsiveness to other antigens. At the other side of the spectrum is operational or "prope" (meaning "almost" or "near") tolerance, which describes an immune state in which only very lowlevel immunosuppression is required to maintain the health of the transplant (11). While specific immune tolerance is the goal of transplant researchers, prope tolerance appears to be a more realistic objective for lung transplant recipients, given the inherent challenges to allograft survival posed by constant environmental exposure, native immunogenicity, and compromised vascular supply.

While a prior study demonstrated that costimulation blockade of CD154/CD40 and $\mathrm{CD} 28 / \mathrm{B} 7$ interactions induces lung transplant acceptance, infection with the common bacterium Psendomonas aeruginosa abolished tolerance (12). Beyond frequent exposure to bacterial pathogens, lung transplant recipients may be especially vulnerable to aerosolized viruses that are able to directly trigger heterologous immunity against donor MHC $(13,14)$. Tolerance induction in the lung has been difficult to recapitulate in animal models of airway and lung transplantation. In murine models, combined therapy targeting CD154 and lymphocyte function-associated antigen 1 (LFA-1), promoted specific immune tolerance in pancreatic islet transplants but failed to do so with airway allografts $(15,16)$. Additionally, mixed chimerism generated through whole body irradiation, bone marrow transplantation, and a brief course of sev- 


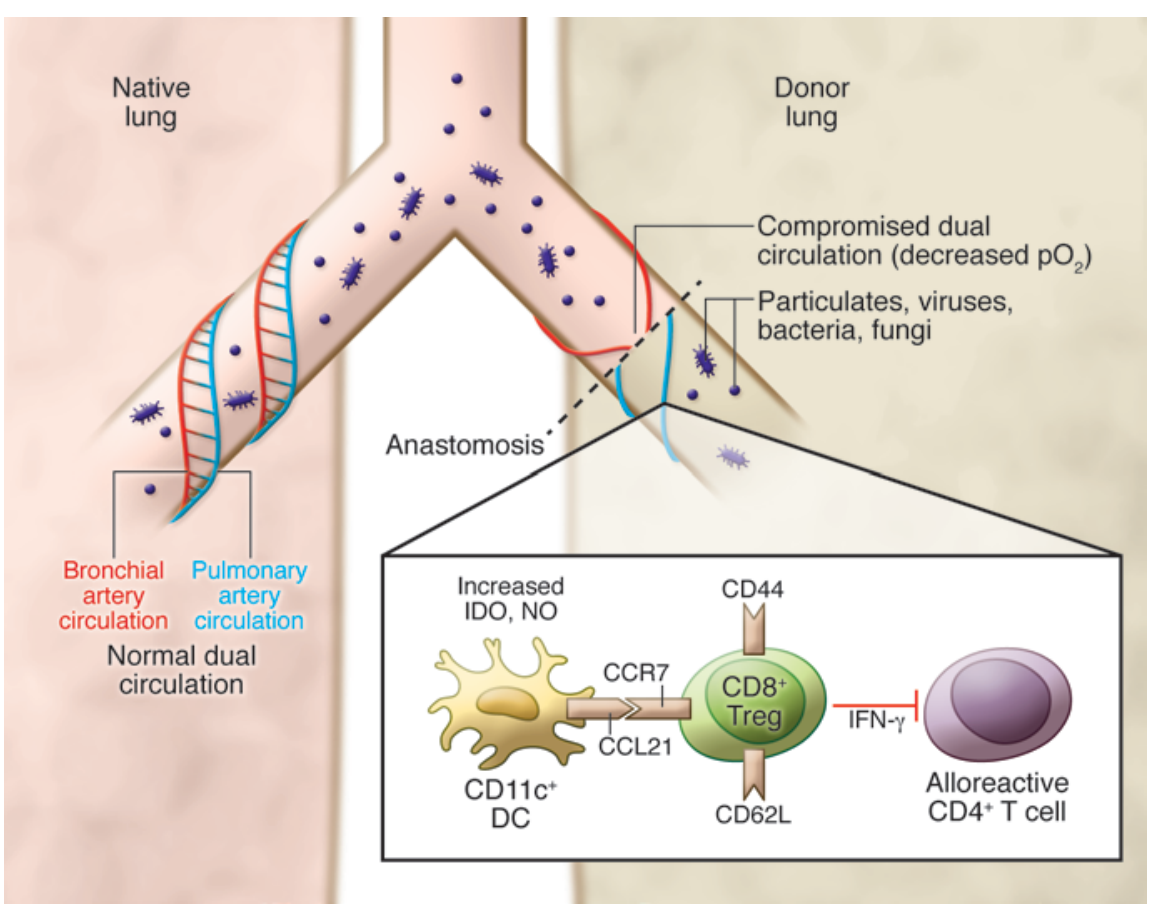

Figure 1

Unique factors promote lung transplant rejection and acceptance. Lung transplants are particularly vulnerable to rejection, due to continual exposure to the external environment. Particulates can trigger inflammation, and pathogens, including viruses, bacteria, and fungi, may promote heterologous immunity. Brain death in the organ donor can cause lung inflammation, which can negatively impact new transplant function. Other factors that contribute to lung allograft rejection are ischemia/reperfusion injury and HLA immunogenicity of the graft. Furthermore, lack of a bronchial artery circulation after surgery may compromise allograft function. While these factors are proinflammatory, lungs also possess unique antiinflammatory properties, including antigen-presenting $\mathrm{CD} 11 \mathrm{c}^{+}$cells that have the capacity to suppress $\mathrm{T}$ cell activation through the increased production of indoleamine 2,3-dioxygenase (IDO). Additionally, Krupnick et al. (10) have described a $\mathrm{CD} 44{ }^{+} \mathrm{CD} 62 \mathrm{~L}+\mathrm{CCR} 7+{ }^{+} \mathrm{CD} 8{ }^{+}$Treg that infiltrates lung transplants and is required for costimulation-induced lung allograft acceptance. The proposed mechanism of CD8 ${ }^{+}$ Treg action is mediated through IFN- $\gamma$ limitation of alloreactive $\mathrm{CD} 4+\mathrm{T}$ cell proliferation and the local upregulation of NO. The chemokine receptor CCR7 on CD8+ Tregs may bind to CCL21 on $\mathrm{CD}_{11 \mathrm{C}^{+}}$dendritic cells to dampen immune responses.

eral immunomodulating therapies led to long- term kidney acceptance but not lung allograft acceptance in a nonhuman primate study (17). Due to the difficulty of blocking rejection and the negative consequence of infection in overly immunocompromised patients, the key objective following lung transplantation should be to specifically target damaging immunity while still preserving protective immunity.

While the International Society for Heart and Lung Transplantation (ISHLT) criteria for rejection has become more specific for delineating different manifestations of acute rejection, the current ISHLT pathologic descriptors lack information on the specific contribution of individual constituents of the immune response in alloimmune injury. The moniker "inflam- mation" is not informative enough to guide therapy reliably, because not all infiltrates are equally damaging. For example, endomyocardial biopsies from heart transplant recipients that contain Quilty lesions, which are collections of $\mathrm{T}$ cells, B cells, macrophages, and plasma cells, do not indicate acute rejection (18). In fact, it is possible that the accumulation of cells in Quilty lesions is actually beneficial to transplant health. A prior preclinical study from D. Kreisel's group demonstrated that lung transplant acceptance occurred following induction of BALT-associated Foxp $3^{+}$cells and recipient $\mathrm{T}$ cells interacting with $\mathrm{CD} 11 \mathrm{c}^{+}$dendritic cells (19). Krupnick et al. extended these findings, demonstrating that a subpopulation of infiltrating $\mathrm{T}$ cells is required for certain immune therapies to create a prope tolerant-like state in the murine lung (10). Furthermore, elimination of this $\mathrm{T}$ cell population was detrimental to the health of the transplant.

\section{A role for $\mathrm{CD}^{+}$Tregs in transplantation}

Most Treg research has focused on the protective function of $\mathrm{CD}^{+}$Tregs in both autoimmune and transplantation tolerance studies. In addition to the well-established participation of $\mathrm{CD}^{+}$Tregs in immune regulation, a prominent and unique role for $\mathrm{CD}^{+}$Tregs has also been established (20). CD8 ${ }^{+}$"suppressor" cells were first described in the 1970s $(21,22)$, but the $\mathrm{CD}^{+}$Treg field lay fallow through the 1980s, due to difficulties in cloning these antigen-specific "suppressor" $\mathrm{T}$ cells and an inability to characterize the biochemical nature of soluble suppressor factors (23). There was a resurgence of interest in the $\mathrm{T}$ cell populations regulating autoimmune and transplant responses in the 1990s, and the pejorative connotation associated with the term "suppressor" $\mathrm{T}$ cells was avoided, as these cells came to be known generally as "regulatory" $\mathrm{CD} 8^{+} \mathrm{T}$ cells, just like their $\mathrm{CD} 4^{+}$Treg counterparts.

In mice, $\mathrm{CD}^{+}$Tregs are subdivided into Qa-1-restricted and non-Qa-1-restricted populations. Qa-1-restricted Tregs recognize the mouse homolog of HLA-E and suppress autoreactive follicular Th cells (24). Non-Qa-1-restricted murine CD8 ${ }^{+}$ Tregs are $\mathrm{CD} 8^{+} \mathrm{CD} 28^{-}$and interfere with antigen presentation through tolerization of dendritic cells via induction of immunoglobulin-like transcript 3 (ILT3) and ILT4 (25). Previously identified $\mathrm{CD}^{+} \mathrm{CCR}^{+}$ Tregs express the classical regulatory marker Foxp3 and rely on interference with very early steps of the TCR signaling cascade to suppress immune responses (26). Unlike $\mathrm{CD}^{+} \mathrm{Foxp}^{+}$Tregs, which are generated in the thymus, generation of $\mathrm{CD}^{+}$ Tregs appears to require antigen stimulation; however, the requirements for $\mathrm{CD}^{+}$ Treg induction (including by alloantigen) remain poorly understood (27). Previous reports have described a protective role for CD8 ${ }^{+}$Tregs in skin, heart, bone marrow, and kidney transplants (28-31).

In a series of elegant experiments performed in a robust acute rejection model, Krupnick and colleagues (10) have discovered a unique population of cells that infiltrates mouse orthotopic lung transplants and is essential for costimulation 
blockade-mediated allograft acceptance (Figure 1). Krupnick et al. discovered that early pulmonary trafficking of these $\mathrm{CD}^{+} \mathrm{CD} 44^{\text {hi }} \mathrm{CD} 62 \mathrm{~L}^{\text {hi }} \mathrm{CCR} 7^{+} \mathrm{T}$ cells, guided by alloantigen specificity, controls rejection responses in an IFN- $\gamma$ - and iNOSdependent manner. IFN- $\gamma$ has previously been demonstrated to be variably required for allograft acceptance, depending on the immunomodulatory therapy and the murine strain combination used in the transplant model (32-34). IFN- $\gamma$ may promote the induction of long-term allograft survival by limiting the proliferation of alloactivated T cells (35). In the Krupnick study, $\mathrm{CD}^{+} \mathrm{T}$ cell-derived IFN- $\gamma$ was important for limiting rejection responses, and iNOS production was required for $\mathrm{CD}^{+}$Tregmediated suppression of $\mathrm{CD}^{+} \mathrm{T}$ cell proliferation. Based on these observations, Krupnick et al. speculate that increased pulmonary allograft NO levels are closely tied to graft survival. However, like IFN- $\gamma$, the role of iNOS in allograft acceptance is probably complex and diverse. For example, pharmacologic inhibition of iNOS and iNOS deficiency in upper airway transplant recipients was actually protective (36), and blocking iNOS prevented injury in a preclinical liver transplant model (37). Once again, the unique site-specific physiology of the lung transplant may explain differences in results among the study by Krupnick and colleagues and studies in other organ transplant models.

Finally, the Kreisel group productively used intravital microscopy to elucidate the impact of CCR7 on $\mathrm{CD}^{+} \mathrm{T}$ cells. There are conflicting reports in the literature about the net effects of blocking CCR7 in transplant. Interestingly, a prior report identified a population of CCR7 ${ }^{+} \mathrm{CD} 8$ Tregs with a naive phenotype, while Krupnick et al. describe CCR7 ${ }^{+}$CD8 Tregs with a central memory phenotype in their lung transplant study (26). It is becoming clear that the array of phenotypes and functions associated with CD8 ${ }^{+}$Tregs is complex. Regardless, the identification of CCR7 as an important receptor for controlling the migration of memory $\mathrm{T}$ cells provided rationale to study the impact of this receptor on recipient-derived $\mathrm{CD}^{+} \mathrm{T}$ cells emigrating into the allograft. Adoptive transfer of $\mathrm{CD}^{+} \mathrm{T}$ cells from $\mathrm{Ccr} 7^{-/-}$mice into $\mathrm{Cd} 8^{-/-}$allograft recipients that also received costimulation blockade resulted in acute transplant rejection; however, this same rejection was not seen in $C d 8^{-/-}$allograft recipients that received $\mathrm{CD} 8^{+} \mathrm{T}$ cells from
WT mice. Two-photon microscopy, which permits real-time imaging of intercellular relations in vivo, revealed that the $\mathrm{CD}^{+}$ T cells lacking CCR7 had very brief interactions with $\mathrm{CD} 11 \mathrm{c}^{+}$dendritic cells compared with WT CD8 ${ }^{+} \mathrm{T}$ cells. This finding suggests that sufficient contact time between $\mathrm{CD}^{+}$Tregs and APCs is required for effective tolerance induction. By evaluating the element of time in the characterization of the immune synapse, Krupnick and colleagues have demonstrated the power of intravital microscopy for characterizing immune tolerance requirements and as a tool for immunology research in general.

\section{Conclusion}

Enhancing host immune regulation, even without generating durable specific immune tolerance, is a worthwhile goal for clinician-scientists. The notion of prope tolerance was advanced originally to explain success achieved in kidney transplantation with the use of Campath-1H (11), an agent which causes a profound deletion of $\mathrm{T}$ cells (including potentially helpful regulatory cells); however, prope tolerance could be a reasonable goal for nondeletional tolerizing therapies in inherently difficultto-tolerize organs, such as the lung. As a more sophisticated understanding of the unique roles played by graft-infiltrating cells is obtained, immunotherapies can be better tailored to the site-specific biology of the transplanted organ.

\section{Acknowledgments}

The authors thank Cornelia Weyand for critically reading this article. The authors' research is supported by NIH grants R01 HL095686 and P01 HL10879701 and VA Merit Award BX000509 (to M.R. Nicolls).

Address correspondence to: Mark R. Nicolls, Associate Professor of Medicine, VA Palo Alto Health Care System, 3801 Miranda Ave., 111P, Palo Alto, California 94304, USA. Phone: 650.493.5000, ext. 69289; Fax: 650.849.0553; E-mail: mnicolls@stanford.edu.

1. Swanson KA, Zheng Y, Heidler KM, Mizobuchi $\mathrm{T}$, Wilkes DS. CDllc + cells modulate pulmonary immune responses by production of indoleamine 2,3-dioxygenase. Am J Respir Cell Mol Biol. 2004;30(3):311-318.

2. Kandaswamy R, et al. A prospective randomized trial of steroid-free maintenance regimens in kidney transplant recipients--an interim analysis. Am J Transplant. 2005;5(6):1529-1536.

3. McDiarmid SV, et al. A randomized prospective trial of steroid withdrawal after liver transplanta- tion. Transplantation. 1995;60(12):1443-1450.

4. Feng $S$, et al. Complete immunosuppression withdrawal and subsequent allograft function among pediatric recipients of parental living donor liver transplants. JAMA. 2012;307(3):283-293.

5. Scandling JD, et al. Tolerance and chimerism after renal and hematopoietic-cell transplantation. NEngl J Med. 2008;358(4):362-368.

6. Kawai T, et al. HLA-mismatched renal transplantation without maintenance immunosuppression. N Engl J Med. 2008;358(4):353-361

7. Nicolls MR, Zamora MR. Bronchial blood supply after lung transplantation without bronchial artery revascularization. Curr Opin Organ Transplant. 2010;15(5):563-567.

8. Prop J, Wildevuur CR, Nieuwenhuis P. Lung allograft rejection in the rat. II. Specific immunological properties of lung grafts. Transplantation. 1985; 40(2):126-131

9. Alwayn IP, Xu R, Adler WH, Kittur DS. Does high MHC class II gene expression in normal lungs account for the strong immunogenicity of lung allografts? Transpl Int. 1994;7(1):43-46.

10. Krupnick AS, et al. Central memory $\mathrm{CD}^{+} \mathrm{T}$ lymphocytes mediate lung allograft acceptance. J Clin Invest. 2014;124(3):1130-1143.

11. Calne $\mathrm{R}$, et al. Prope tolerance, perioperative campath $1 \mathrm{H}$, and low-dose cyclosporin monotherapy in renal allograft recipients. Lancet. 1998; 351(9117):1701-1702

12. Yamamoto S, et al. Cutting edge: Pseudomonas aeruginosa abolishes established lung transplant tolerance by stimulating B7 expression on neutrophils. J Immunol. 2012;189(9):4221-4225.

13. Adams AB, et al. Heterologous immunity provides a potent barrier to transplantation tolerance. J Clin Invest. 2003;111(12):1887-1895.

14. Chen HD, Fraire AE, Joris I, Welsh RM, Selin LK. Specific history of heterologous virus infections determines anti-viral immunity and immunopathology in the lung. Am J Pathol. 2003;163(4):1341-1355.

15. Nicolls MR, Coulombe M, Beilke J, Gelhaus HC, Gill RG. CD4-dependent generation of dominant transplantation tolerance induced by simultaneous perturbation of CD154 and LFA-1 pathways. Jimmunol. 2002;169(9):4831-4839.

16. Murakawa T, et al. Simultaneous LFA-1 and CD40 ligand antagonism prevents airway remodeling in orthotopic airway transplantation: implications for the role of respiratory epithelium as a modulator of fibrosis. J Immunol. 2005; 174(7):3869-3879.

17. Aoyama A, et al. Comparison of lung and kidney allografts in induction of tolerance by a mixedchimerism approach in cynomolgus monkeys. Transplant Proc. 2009;41(1):429-430.

18. Tan CD, Baldwin WM, Baldwin WM 3rd, Rodriguez ER. Update on cardiac transplantation pathology. Arch Pathol Lab Med. 2007;131(8):1169-1191.

19. Li W, et al. Lung transplant acceptance is facilitated by early events in the graft and is associated with lymphoid neogenesis. Mucosal Immunol. 2012;5(5):544-554.

20. Chess L, Jiang H. Resurrecting CD8+ suppressor T cells. Nat Immunol. 2004;5(5):469-471.

21. Cantor H, Shen FW, Boyse EA. Separation of helper T cells from suppressor T cells expressing different Ly components. II. Activation by antigen: after immunization, antigen-specific suppressor and helper activities are mediated by distinct $\mathrm{T}$-cell subclasses. J Exp Med. 1976;143(6):1391-1340.

22. Jandinski J, Cantor H, Tadakuma T, Peavy DL, Pierce CW. Separation of helper T cells from suppressor $\mathrm{T}$ cells expressing different Ly components. I. Polyclonal activation: suppressor and helper activities are inherent properties of distinct T-cell subclasses. J Exp Med. 1976;143(6):1382-1390.

23. Kapp JA, Bucy RP. CD8+ suppressor T cells resurrected. Hum Immunol. 2008;69(11):715-720. 
24. Kim HJ, Verbinnen B, Tang X, Lu L, Cantor H. Inhibition of follicular T-helper cells by CD8(+) regulatory $\mathrm{T}$ cells is essential for self tolerance. Nature. 2010;467(7313):328-332.

25. Manavalan JS, et al. High expression of ILT3 and ILT4 is a general feature of tolerogenic dendritic cells. Transpl Immunol. 2003;11(3-4):245-258.

26. Suzuki M, et al. CD8+CD45RA+CCR7+FOXP3+ T cells with immunosuppressive properties: a novel subset of inducible human regulatory $\mathrm{T}$ cells. J Immunol. 2012;189(5):2118-2130.

27. Suzuki M, Konya C, Goronzy JJ, Weyand CM. Inhibitory CD8+ T cells in autoimmune disease. Hum Immunol. 2008;69(11):781-789.

28. Li XL, et al. Mechanism and localization of CD8 regulatory $\mathrm{T}$ cells in a heart transplant model of tolerance. J Immunol. 2010;185(2):823-833.

29. Beres AJ, Haribhai D, Chadwick AC, Gonyo PJ,
Williams CB, Drobyski WR. CD8+ Foxp3+ regulatory $\mathrm{T}$ cells are induced during graft-versus-host disease and mitigate disease severity. J Immunol. 2012;189(1):464-474

30. Lerret NM, Houlihan JL, Kheradmand T, Pothoven KL, Zhang ZJ, Luo X. Donor-specific CD8+ Foxp3+ $T$ cells protect skin allografts and facilitate induction of conventional CD4+ Foxp3+ regulatory T cells. Am J Transplant. 2012;12(9):2335-2347.

31. Zhou J, Carr RI, Liwski RS, Stadnyk AW, Lee TD. Oral exposure to alloantigen generates intragraft CD8+ regulatory cells. J Immunol. 2001;167(1):107-113.

32. Nicolls MR, Coulombe M, Bolwerk A, Beilke J, Gill RG. IFN- $\gamma$ is not a universal requirement for allograft survival. Transplantation. 2002;74(4):472-477.

33. Markees TG, et al. Long-term survival of skin allografts induced by donor splenocytes and antiCD154 antibody in thymectomized mice requires
CD4(+) T cells, interferon- $\gamma$, and CTLA4. J Clin Invest. 1998;101(11):2446-2455.

34. Konieczny BT, et al. IFN- $\gamma$ is critical for long-term allograft survival induced by blocking the CD28 and CD40 ligand $\mathrm{T}$ cell costimulation pathways. J Immunol. 1998;160(5):2059-2064.

35. Hassan AT, et al. Regulation of alloantigen-mediated T-cell proliferation by endogenous interferon- $\gamma$ : implications for long-term allograft acceptance. Transplantation. 1999;68(1):124-129.

36. Minamoto K, Pinsky DJ. Recipient iNOS but not eNOS deficiency reduces luminal narrowing in tracheal allografts. J Exp Med. 2002;196(10):1321-1333.

37. Shi Y, Rehman H, Wright GL, Zhong Z. Inhibition of inducible nitric oxide synthase prevents graft injury after transplantation of livers from rats after cardiac death. Liver Transpl. 2010; 16(11):1267-1277.

\title{
Neurogenesis or non-neurogenesis: that is the question
}

\author{
Gianvito Martino, Erica Butti, and Marco Bacigaluppi
}

Neuroimmunology Unit, Institute of Experimental Neurology (INSpe), Division of Neuroscience, San Raffaele Scientific Institute, Milan, Italy.

\begin{abstract}
Neural stem/precursor cells (NPCs) that reside within germinal niches of the adult CNS have more complex roles than previously expected. In addition to their well-documented neurogenic functions, emerging evidence indicates that NPCs exert non-neurogenic functions that contribute to the regulation and preservation of tissue homeostasis under both physiological and pathological conditions. In this issue of the JCI, Mohammad et al. found that DCs efficiently patrol the CNS only when the germinal niche of the subventricular zone functions properly. Indeed, DCs traveled from the ventricles along the rostral migratory stream to the olfactory bulb (a cervical lymph node access point) to dampen anti-CNS immune responses. The authors' findings further support a non-neurogenic role for NPCs in maintaining tissue homeostasis and promoting tissue protection in the adult brain.
\end{abstract}

\section{Neurogenesis: A tale of two germinal niches}

In the adult rodent CNS, lifelong neurogenesis - the process of neuron generation from neural stem/progenitor cells (NPCs) - primarily occurs in two distinct areas of the brain (i.e., germinal niches), the subgranular zone (SGZ) of the hippocampus and the subventricular zone (SVZ) of the lateral ventricles $(1,2)$. Depending on the germinal niche, NPCs have distinct fates. Adult NPCs generated in the SGZ migrate a short distance into the granule cell layer of the dentate gyrus (DG) and become indistinguishable from preexisting cells, an activity that is considered necessary for

Conflict of interest: The authors have declared that no conflict of interest exists.

Citation for this article: J Clin Invest. 2014;

124(3):970-973. doi:10.1172/JCI74419. modulating and refining the neuronal circuits involved in hippocampus-dependent memory processing and behavior (1-3). Newly formed NPCs from the SVZ migrate along the rostral migratory stream (RMS) to the olfactory bulb (OB), where they integrate within the granule and glomerular cell layers to maintain and reorganize the OB system $(1,2)$. Recent compelling evidence challenges the limited view that neurogenic areas of the brain act solely as sources of newly formed neurons for replacement of neuronal cells in the hippocampus and $\mathrm{OB}(4)$. In fact, the exclusive neurogenic role of the SVZ has been questioned due to recent data clearly indicating that adult $O B$ neurogenesis might not have any functional significance in humans. In adult humans, 700 new neurons are added to the hippocampus each day (corresponding to an annual turnover of $1.75 \%$ of the neurons within the renewing fraction); however, retrospective birth dating has established that the majority of $\mathrm{OB}$ neurons are of the same age as the individual, and that additional neurons in the adult human OB account for less than $1 \%$ of the total neurons exchanged over a century (4).

\section{Not only for neurogenesis: SVZ-derived NPCs exhibit non-neurogenic functions}

How can we explain the apparent paradox of NPCs being produced by the SVZ, yet no evident neuron turnover in the $\mathrm{OB}$ ? One thought-provoking explanation comes from recent studies indicating that adult NPCs residing within the SVZ exert non-neurogenic functions - such as protecting and regulating homeostasis - as alternatives to cell replacement, in both physiological and pathological conditions (Table 1 and Figure 1). For example, it has been shown that SVZ-derived NPCs have phagocytic activity toward maturing neurons, which requires the intracellular engulfment protein ELMO1 to promote Rac activation downstream of phagocytic receptors (5). Additionally, SVZ-derived NPCs have been described as having a secretory protein profile (including secretion of VEGF) distinct from other brain cells and capable of modulating activation, proliferation, and phagocytosis of microglia (6). 